\title{
Endogenous retroviruses transcriptomes in response to four avian pathogenic microorganisms infection in chicken
}

\section{Manman Dai}

South China Agricultural University

\section{Tingting Xie}

South China Agricultural University

\section{Min Feng}

South China Agricultural University

Xiquan Zhang ( $\nabla$ xqzhang@scau.edu.cn)

College of Animal Science in South China Agriculture University. Guangdong Provincial Key Lab of AgroAnimal Genomics and Molecular Breeding and Key Laboratory of Chicken Genetics, Breeding and reproduction, Ministry of Agriculture.

\section{Research}

Keywords: endogenous retroviruses, chicken, pathogenic microorganism, ERV transcriptome

Posted Date: April 16th, 2020

DOl: https://doi.org/10.21203/rs.3.rs-22243/v1

License: (9) This work is licensed under a Creative Commons Attribution 4.0 International License. Read Full License 


\section{Abstract}

\section{Background}

Endogenous retroviruses (ERVs) are common in the genomes of vertebrates and have been implicated in a variety of host responses such as immunity, phenotypic character and disease occurrence. However, the impact of ERVs on chicken disease is not well understood. In particular, the expression profiles of chicken ERVs (ChERVs) in the infection of pathogenic microorganisms are not clear.

\section{Results}

In the present study, we systematically identified 436 full-length ChERVs from the chicken genome and analyzed their neighboring genes. Subsequently, ChERV transcriptomes were analyzed in chicken after subgroup $\mathrm{J}$ avian leukosis virus (ALV-J), avian influenza virus (AIV), Marek's disease virus (MDV) and avian pathogenic escherichia coli (APEC) infection. We found that about $50 \%-68 \%$ of ChERVs were transcriptionally active in infected-samples and uninfected-samples, although the abundance of most ChERVs is relatively low. Moreover, compared to uninfected-samples, a few ChERVs were significantly differentially expressed in ALV-J (49 ChERVs), AIV (18 ChERVs), MDV (66 ChERVs) and APEC (17 ChERVs) infected-samples. Of these differentially expressed ChERVs, only ChERV-3 was simultaneously down-regulated in the four ERV transcriptomes analysis after ALV-J, AIV, MDV and APEC infection. Further verification experiments found that both the exogenous and endogenous expression of ChERV-3 env gene was inhibited after ALV-J infection in chicken fibroblasts. However, overexpression of ChERV-3 env could induce the expression of interferon-stimulated genes (ISGs), but did not affect the replication of ALV-J.

\section{Conclusions}

To our knowledge, this study systematically revealed the expression profile of ChERVS for the first time after the pathogenic microorganism infection in chicken. These findings may be of significance for understanding the role and function of ChERVs to response the pathogenic microorganism infection.

\section{Background}

Endogenous retroviruses (ERVs) are integrated retroviral elements that are present in a wide range of vertebrate genomics, including chickens $[1,2]$. ERVs contain three main coding genes: gag, pol and env, flanked by long terminal repeats (LTRs), which are control regions containing promoters, enhancers and polyadenylation signals [1]. Initially considered as "junk DNA", ERVs have been shown to be involved in many host biological processes such as placental development [3], cancers [4], autoimmune diseases [5], antiviral immunity [6] and reproduction [7]. However, even in human research, the impact of ERVs on human health and disease is still not well understood.

The study of chicken ERV (ChERV) can be traced back to the beginning of ERV discovery to the present. ChERV is one of the 3 types of ERV originally discovered at around the same time in the late 1960s and 
early 1970s [8]. Subsequent research found that ChERV plays an important role in both qualitative and quantitative traits as follows. ChERVs have been found to be involved in blue eggshell $[9,10]$, recessive white [11], late feathering [12] and henny feathering phenotype [13]. In addition, the effects of ChERVs on exogenous avian tumor viruses infection including leukosis virus (ALV), reticuloendotheliosis viruses (REV) and Marek's disease virus (MDV) have also been documented [14-17]. Still, the contribution of ChERVs to chicken disease is less understood and understudied. Additionally, there have been no reports of ChERV affecting diseases caused by infection with other avian pathogenic microorganisms except for tumor viruses.

Accordingly, in this study, we systematically analyze the potential functions of ChERVs in the infection of pathogenic microorganisms such as subgroup $\mathrm{J}$ avian leukosis virus (ALV-J), and avian influenza virus (AIV), MDV and avian pathogenic escherichia coli (APEC) that cause chicken diseases. It is well documented that ERVs can affect the host responses at multiple levels. For instance, LTR of ERVs can modulate the expression of adjacent host genes in genome [18]. The expression of ERV proteins with conventional retroviral functions can influence the host biological process $[14,19,20]$. Moreover, noncoding RNA produced from ERVs also has been found to be biologically active [21, 22]. Given that both complex biological mechanisms and technical challenges, deciphering ERVs biological function is far from being simple. In all cases, it is relatively easy to explore the function of ERV by analyzing the expression level of ERVs. Thus, ChERV transcriptomes were analyzed after ALV-J, AIV, MDV and APEC infection in chicken using the published data [23-26]. We provide an overview of the expression of ChERVs in the chicken tissues of ALV-J, AIV, MDV and APEC infection. Furthermore, our results also provide insights into the interaction between a specific ChERV and ALV-J, and may be of significance for elucidating the biological functions of ChERVs.

\section{Methods}

\section{Identification and annotation of chicken ERVs}

The chicken genome (Gallus_gallus-5.0) was used as the input for ChERVs identification with two pipelines (Fig. 1A). In the first pipeline, ChERVs were screened in the silkworm genome using the software LTRharvest (https://www.zbh.uni-hamburg.de/LTRharvest) [27] and LTRdigest (http://bigd.big.ac.cn/biocode/tools/BT003054) [28]. Pairs of putative LTRs that were separated by 1 to $15 \mathrm{~kb}$ and flanked by target site duplications (TSD) were screened by LTRharvest. The threshold of LTR nucleotide similarity used in LTRharvest was greater than $80 \%$, and other parameters were set to their defaults. Internal sequence retroviral features of ERV candidates, including protein domains, polypurine tracts (PPT), and primer-binding sites (PBS), were annotated using LTRdigest with default parameters. A second pipeline, MGEScan-LTR (https://sourceforge.net/projects/mgescan/) was also employed to identify ChERVs using the default parameters. Each candidate containing at least three of the five canonical retroviral protein domains (Gag, PR, RT, IN, and RH) was retained in the results from LTRharvest and MGEScan-LTR. Finally, the ERV candidates identified by the two pipelines were merged as the ChERV library. 
Sequences of the RT domain from ChERVs and known exogenous and endogenous retroviruses [29] were used for multiple alignment using MUSCLE (v3.8.31) (https://www.biostars.org/p/185419/). A neighborjoining phylogeny was built from the RT domain alignment using MEGA6 (https://www.megasoftware.net) with 1,000 bootstrap replicates. The putative families of BmERVs were defined based on their support in phylogenetic trees.

\section{Identification of genes located in the neighborhood of ERVs in the chicken genome}

To show the distribution of ChERVs on the chromosome, we display ChERVs on each chromosome of the chicken. Moreover, the UCSC Genome Bioinformatics tool (http://pgb.ibe.upf.edu) was used to screen for genes located within $100 \mathrm{~kb}$ of upstream and downstream of ChERVs (Fig. 1A). Target genes are defined as genes that have annotated exons (UTR and CDS) within the defined sequence space of $100 \mathrm{~kb}$. Blast2GO (https://www.blast2go.com) and WEGO (http://wego.genomics.org.cn) were used to perform gene ontology (GO) classification. The GO terms included molecular function, cellular component and biological process. For the pathway enrichment analysis, the genes were mapped to Kyoto Encyclopedia of Genes and Genomes (KEGG) database (https://www.kegg.jp/kegg/). The interaction networks of ChERVs and neighboring immune-related genes were imported to Cytoscape software (https://cytoscape.org) for visualization.

\section{Four Transcriptome Raw Data Of Chicken Pathogenic Microorganism}

To analyze the ChERVs transcriptome in chicken infected with pathogenic microorganism including AIV, ALV-J, MDV and APEC (Fig. 1A), we downloaded the raw reads from NCBI with the details as follows.

In the study that provided the transcriptome raw data of AIV infection for our analysis [24], H5N1-infected chicken ileum samples and PBS-infected ileum samples (3 samples from each of 1 day post infection (dpi) were selected for the analysis of ChERVs transcriptome. These six CDNA libraries were designated as GGA_HP_ileum_1_rep1 (SRA ID: ERR597332), GGA_HP_ileum_1_rep2 (ERR597329), GGA_HP_ileum_1_rep3 (ERR597323), GGA_con_ileum_1_rep1 (ERR597325), GGA_con_ileum_1_rep2 (ERR597328), GGA_con_ileum_1_rep3 (ERR597319).

The transcriptome raw data of ALV-J-infected spleen sample and control sample come from our previous study and download from GEO (accession: GSE63226) [23]. Two cDNA libraries of ALV-J infected and uninfected samples from 140-day-old female chickens of White Recessive Rock were designated as WRR+ (GSM1544045) and WRR- (GSM1544046).

The published splenic transcriptome raw data of MDV-infected samples and uninfected samples at 14 dpi were selected for ChERVs transcriptome analysis [25]. Two cDNA libraries of MDV-infected and uninfected samples were designated as $\mathrm{CH}-14 \mathrm{dpi}$ (SRA:SRX2425016) and $\mathrm{CH}-14 \mathrm{~d}$ (SRA:SRX2425017). 
The published bursa of fabricius transcriptome raw data of APEC-infected samples and uninfected samples at 5 dpi were selected for ChERVs transcriptome analysis [26]. Seven cDNA libraries of APECinfected and uninfected samples of susceptible phenotype chickens were designated as bursa_D5_S_rep1(GSM1724128), bursa_D5_S_rep2(GSM1724129), bursa_D5_S_rep3(GSM1724130), bursa_D5_S_rep4(GSM1724131), bursa_D5_NC_rep1(GSM1724113), bursa_D5_NC_rep2(GSM1724114) and bursa_D5_NC_rep3(GSM1724115) (4 per each infected group, and 3 per each uninfected group).

\section{Analysis Of Transcriptome Raw Data}

To acquire high quality clean reads, the raw reads were filtered by removing the adapter-containing reads and low quality reads. The remaining clean reads were mapped to the chicken genome assembly (Gallus_gallus-5.0) using TopHat2 (version 2.0.3.12) (http://ccb.jhu.edu/software/tophat/index.shtml) [30]. The mapped reads of each sample were assembled by software Cumerge (https://www.biostars.org/p/44302/), TopHat2 and Cufflinks (http://cole-trapnell-lab.github.io/cufflinks/) [31]. Genes and ChERVs abundances were quantified by software RSEM (http://deweylab.github.io/RSEM/) [32] and their expression level was normalized by FPKM (Fragments Per Kilobase of transcript per Million mapped reads).

To identify differentially expressed ChERVs (DEEs) and differentially expressed genes(DEGs), the edgeR package (http://www.rproject.org/) was used. ChERVs and genes with fold change of $|\log 2 \mathrm{FC}| \geq 1$ (FC: fold change) and a false discovery rate (FDR) $<0.05$ were considered as DEEs and DEGs.

\section{Effect Of ALV-J Infection On ChERV-3 Expression}

To demonstrate the effect of ALV-J infection on ChERV-3 expression, quantitative real-time PCR (qPCR) was used to detect the endogenous expression of ChERV-3 env gene after ALV-J (SCAU-HNO6, $10^{5}$ $\mathrm{TCID}_{50} / \mathrm{mL}$ ) infection at 24 and $48 \mathrm{~h}$ post infection (hpi) in primary chicken embryo fibroblasts (CEF). Uninfected CEF was used as control. The qPCR primer of ChERV-3 env were designed using the NCBI Primer BLAST program (F: TGTCAGCGGATGTTGTGGAA; R: CATCCAGGTGTGAGGTGCTT). The GAPDH gene was used as an internal control. qPCR was performed on a Bio-Rad CFX96 Real-Time Detection System using iTaqTM Universal SYBR® Green Supermix Kit reagents (Bio-Rad, CA, USA) according to the manufacturer's specifications. Data analyses were performed using the $2^{-\triangle \Delta C t}$ method.

To further explore whether ALV-J infection affects the exogenous expression of ChERV3 envelope protein, we synthesized the $3 x$ flag-ERV3env sequence (Additional file 1) by gene synthesis and inserted into pcDNA3.1 vector by $5^{\prime} \mathrm{Kpnl}$ and 3' BamH to construct the pcDNA3.1-ERV3env plasmid. DF1 cells, belong to chicken embryo fibroblast cell lines, are known to be susceptible only to exogenous ALV [33] and obtained from ATCC (Manassas, USA). DF1 cells were cultured in 24-well plates and transfected with $0.75 \mu \mathrm{g}$ pcDNA3.1-ERV3env plasmid using Lipofectamine 3000 reagent (ThermoFisher, USA). $24 \mathrm{~h}$ later, the transfected DF1 cells were infected with $10^{5} \mathrm{TCID}_{50} / \mathrm{mL}$ of ALV-J strain SCAU-HN06. Uninfected DF1 
cells transfected with pcDNA3.1-ERV3env plasmid were used as control. The exogenous expression of ChERV3 env mRNA was analyzed by qPCR and the envelope protein was analyzed by Western blot using flag antibody (Beyotime, China) at 24 and $48 \mathrm{hpi}$. Data are representative of three independent experiments, which are performed in triplicate.

\section{Overexpression of ChERV-3 env gene and measurement of ALV-J infection in DF1cells}

On the other hand, DF1 cells were transfected with $0.75 \mu \mathrm{g}$ pcDNA3.1-ERV3env plasmid and pcDNA3.1 empty vector (NC), respectively. These transfected DF1 cells were infected with $10^{5} \mathrm{TCID}_{50} / \mathrm{mL}$ of ALV-J strain SCAU-HN06 at $24 \mathrm{~h}$ post transfection. qPCR was employed to detect the production of ALV-J in mRNA level with the specific primers of ALV-J gp85 gene [34]. The cell supernatants were tested for ALV group-specific antigen (p27) using the Avian Leukosis Virus Antigen ELISA Test Kit (Zoetis, USA) according to the manufacturer's instructions. The results were expressed as $s / p$ ratios where $s / p=$ (Sample Mean-Kit Negative Control Mean) / (Kit Positive Control Mean-Kit Negative Control Mean). All experiments were performed in triplicate.

\section{Analysis Interferon-stimulated Genes (ISGs) By qPCR}

To analyze the effect of ChERV3 envelope protein on host antiviral factors, DF1 cells were transfected with $0.75 \mu \mathrm{g}$ pcDNA3.1-ERV3env plasmid and pcDNA3.1 empty vector, respectively using Lipofectamine 3000 (Invitrogen, USA). Chicken interferon-stimulated genes (ISGs) including STAT1, EIF2AK2(PKR), ISG12, $\mathrm{Mx}$ and $\mathrm{CH} 25 \mathrm{H}$ were detected by qPCR at $24 \mathrm{~h}$ post transfection. The GAPDH gene was used as an internal control. All experiments were performed in triplicate.

\section{Statistical Analyses}

Statistical comparisons were performed using GraphPad Prism 5 (GraphPad Software Inc., USA). Results are presented as means \pm SEM, and statistical significance was assessed at $P<0.05(*), 0.01(* *)$, or $0.001(\star \star \star)$.

\section{Results}

\section{De novo detection of ChERVs in the chicken genome}

Two different pipelines were employed for mining ChERVs in the chicken genome 5.0 (Fig. 1). Firstly, ChERVs were detected by LTRharvest and annotated with LTRdigest. A total of 17,353 pairs of putative LTRs in the chicken genome were identified with LTRharvest. These putative LTR pairs were filtered down to 337 ERV candidates by LTRdigest. Additionally, 99 unique ERV candidates were revealed by MGEScan. After merging the ERV candidates identified by the two pipelines, we identified a total of 436 ChERV 
candidates in the chicken genome (Fig. 1A, Additional file 2: Table S1). The full lengths of these 436 ChERV candidates range from 2,555 to $21,180 \mathrm{bp}$, and their LTR lengths vary from 104 to $1781 \mathrm{bp}$.

To classify the detected ChERVs, the RT domain sequences were used to build a multiple alignment and compute phylogenetic trees using the neighbor-joining method implemented in MEGA 6 . Among the 436 ChERVs identified in this study, 384 ChERVs have a RT domain conserved enough to be aligned confidently for phylogenetic analysis. According to the phylogenetic tree analysis, ChERVs were classed into three groups and 15 families, denoted group I to III and families GGERV1 to 15 (Fig. 1B and Additional file 3: Table S2). Most of the ChERVs (247) included in the phylogenetic analysis belong to the group III and were classified into eight families including GGERV11 to GGERV15 (Fig. 1B). Group II ChERV contains the most ERV families ranging from GGERV 3 to GGERV10 comprised of 126 elements (Fig. 1B). Only 11 ChERVs in the GGERV1 and GGERV2 belong to the group I (Fig. 1B). Additionally, 52 ChERVs were marked as unclassified members (Additional file 3: Table S2).

\section{Identification and analysis of (potentially cis-Target) genes that occur in the neighborhood of ChERVs}

Mapping 436 ChERV candidates to chicken chromosomes, we found that a lot of ChERVs are inserted on chromosome 1, 2, 3, 4 and sex chromosome Z, W (Fig. 1C). Especially, ChERVs insertion on the W chromosome is very high (Fig. 1C). Furthermore, to identify host genes that may be regulated by ChERVs, we analyzed the positional relationship between ChERVs and their neighboring genes. A total of 414 encoding genes, 223 long non-coding RNA (IncRNA) and 60 non-coding RNA (ncRNA) such as miRNA and snRNA were identified within $100 \mathrm{~kb}$ of upstream and downstream of these 436 ChERVs (Additional file 4 : Table S3).

The 414 encoding genes were identified as potential cis-target genes of 202 ChERVs. GO and KEGG were employed to further explore the potential functions of these genes. GO biological process analysis showed that most of the candidate cis-target genes were enriched in "cellular process", "developmental process", "single-organism process", "immune system process", "response to stimulus", "metabolic process", and etc (Fig. 1D, Additional file 5: Table S4). KEGG analysis illustrated mainly enrichment in "Ubiquinone and other terpenoid-quinone biosynthesis", "Focal adhesion”, "Ubiquitin mediated proteolysis", "FoxO signaling pathway", "VEGF signaling pathway", and "RIG--like receptor signaling pathway" (Fig. 1E, Additional file 5: Table S4). Based on the analysis of GO and KEGG, 19 immune-related genes such as TRAF6, IL7R, TRAF3IP1, MAPK12 were identified within $100 \mathrm{~kb}$ of upstream and downstream of 17 ChERVs (Additional file 6: Fig. S1, Additional file 5: Table S4).

\section{Transcriptome Analysis of ChERVs in ALV-J, AIV, MDV and APEC infection}

To obtain global view of ChERVs expression after pathogenic microbial infection in chicken, four published transcriptome raw data about ALV-J, AIV, MDV and APEC infection were used to match Gallus_gallus-5.0 genome for deep analysis [23-26]. The analysis resulted in the identification of 258 (59.4\%) ChERVs that were transcribed in ALV-J-infected spleens or their controls (Fig. 2A, Additional file 7: Table S5). Compared to uninfected sample, the expression levels of 15 ChERVs were significantly up- 
regulated and 34 ChERVs were significantly down-regulated in ALV-J-infected spleen (Fig. 2B, Additional file 8: Table S6). 299 (68.6\%) ChERVs were detected to be transcribed in H5N1-infected chicken ileum samples and PBS-infected ileum samples at $1 \mathrm{dpi}$ (Fig. 2C, Additional file 7: Table S5). Scatter plot showed that only 3 ChERVs were significantly up-regulated and 15 ChERVs were significantly downregulated in H5N1-infected chicken ileum samples (Fig. 2D, Additional file 8: Table S6). The transcriptional profiling of ChERVs in MDV-infected spleen and uninfected spleen at 14 dpi showed that 240 (55.0\%) ChERVs were transcribed (Fig. 2E, Additional file 7: S5). Of these 240 ChERVs, 23 ChERVs were significantly up-regulated and $43 \mathrm{ChERVs}$ were significantly down-regulated in MDV-infected spleen samples (Fig. 2F, Additional file 8: Table S6). 219 (50.2\%) ChERVs were detected to be transcribed in APEC-infected bursa samples and uninfected samples at 5 dpi (Fig. 2G, Additional file 7: Table S5). Further analysis showed that the expression of 15 ChERVs were significantly up-regulated and 2 ChERVs were significantly down-regulated in APEC-infected bursa (Fig. 2H, Additional file 8: Table S6). From the results of ChERV transcriptomes analysis in the present study, the expression levels of most of the ChERVs are very low (Fig. 2).

For the differentially expressed ChERVs, the venn diagram showed that only one common ChERV was in the four ERV transcriptomes analysis after ALV-J, AIV, MDV and APEC infection (Fig. 3A). This common ERV was numbered ChERV-3 in this study. Coincidentally, heat map showed that ChERV3 was significantly down-regulated after ALV-J, AIV, MDV and APEC infection (Fig. 3B). Furthermore, the predicted ChERV-3 sequence was used to perform phylogenetic analysis with the verified chicken endogenous retroviruses including ev1, ev3, SD0501, EAV-HP, ALVE-B9/10/11, MLV-related ChERV and Ovex1. Phylogenetic tree showed that ChERV-3 was closely related to ev1, ev3, SD0501and ALVEB9/10/11 (Fig. 3C). By comparing with the sequences of these known chicken endogenous retroviruses, we further determined the exact information of ChERV-3 (Fig. 3D). ChERV-3 has a complete retroviral structure with a length of 7,524 bp and is located on chromosome 1 (Fig. 3D).

\section{The expression of ChERV-3 envelope protein was inhibited after ALV-J infection}

To verify whether ChERV-3 expression was inhibited after infection with pathogenic microorganisms, ALV$J$ was selected for further in vitro experiments. Figure 4A showed that the endogenous expression of ChERV-3 env gene was significantly inhibited after ALV-J infection at 24 and 48 hpi. Furthermore, ChERV3 env gene was synthesized and transfected into DF1 cells. After $24 \mathrm{~h}$ post transfection (hpt), DF1 cells were infected by ALV-J. Compared to the uninfected cells, the qPCR result showed that the exogenous ChERV-3 env expression was significantly inhibited by ALV-J infection at $24 \mathrm{hpi}$ (48 hpt) and $48 \mathrm{hpi} \mathrm{(72}$ hpt) (Fig. 4B). Furthermore, at the protein level, the exogenous expression of ChERV-3 envelope protein was also suppressed after ALV-J infection at the $24 \mathrm{hpi}$ (48 hpt) and $48 \mathrm{hpi} \mathrm{(72} \mathrm{hpt)} \mathrm{(Fig.} \mathrm{4C).}$

\section{ChERV-3 Envelope Protein Has No Effect On ALV-J Production}

Given that the expression of ChERV-3 envelope protein is inhibited by ALV-J infection, we speculate whether the envelope protein of ChERV-3 affects ALV-J replication. Compared to the empty vector control, transfection of ChERV-3 env did not affect ALV-J replication in DF1 cells at 24 and 48 hpi (Fig. 5A, B). 
However, the expression levels of all the ISGs detected in our study were significantly up-regulated in the ChERV-3 env transfected DF1 cells compared with the empty vector transfected group at $24 \mathrm{hpt}$ (Fig. 5C).

\section{Discussion}

ERVs make up a large fraction of host genome, yet the relevance of ERVs in biology is only beginning to be uncovered. In the present study, to explore the role of ChERVs in the process of viruses and bacteria infection in the chicken, we pay particular attention to the transcriptome of ChERVs and their neighboring immune-related genes.

ERVs can affect neighboring genes to produce specific phenotypes. Chicken phenotypes such as blue eggshell, recessive white and late feathering have been demonstrated to be caused by the insertion of ERVs $[9,11,12]$. The LTR sequences of ERV element contain an internal promoter and some regulatory sequences. Therefore, ERVs have the potential to regulate the expression of neighboring genes. In total, 414 encoding genes were identified as neighboring genes of 202 ChERVs in our study. However, only 19 of these 414 coding genes are immune-related. Further investigation revealed that the expression of these immune-related genes were not significantly up-regulated after the ALV-J and AIV infection (Additional file 9: Table S7). SLC39A10 and HIF1A, neighboring immune-related genes of ChERV-361 and ChERV-275, were significantly up-regulated after MDV infection (Additional file 9: Table S7). The expression of ChERVs' neighboring immune-related genes including S1PR1, SLC39A10, TRAF6, VEGFC, IL 7R, CAV1 and MET were significantly up-regulated after APEC infection (Additional file 9: Table S7). These ChERVs' neighboring immune-related genes such as SLC39A10, TRAF6, and IL7R have been found to play an important role in host immune responses [35-37]. However, there is no further reports indicating that the function of these immune-related genes is related to ERVs. In fact, human ERVs could indeed be integrated within gene immunity pathways [38]. Our study only provides clues that immune-related genes may be regulated by ChERVs and then function in MDV and APEC infection. A better understanding of the regulatory relationship based on the position of ChERVs and immune-related genes after MDV and APEC infection would merit further investigations.

Previous study has found that many ChERVs have the ability to transcribe and translate [39]. Here, we used the RNA-seq data to provide a first overview of the ChERV transcriptome in ALV-J, AIV, MDV and APEC infection. We observed that about $50 \%-68 \%$ of ChERVs were transcriptionally active in infectedsamples and uninfected-samples, although the abundance of most of the ChERVs is relatively low. It is well accepted that host genomes have evolved multiple strategies of defense against ERVs involving both epigenetic modifications that restrict ERVs transcription, and host restriction factors that target other phases of the ERVs replication cycle [40-43]. Therefore, more attention should be paid to those ChERVs with high expression abundance, such as ChERV-3, ChERV-304, ChERV-433, and etc.

In order to more directly find out the ChERVs that function in the process of pathogenic microorganism infection in chickens, we have used a common transcriptome analysis strategy, which is to find differentially expressed ERVs. Compared to uninfected-samples, only a few ChERVs were significantly 
differentially expressed in ALV-J (49), AIV (18), MDV (66) and APEC (17) infected-samples (Fig. 2). We found an interesting phenomenon that the number of significantly down-regulated ChERVs was higher than that of significantly up-regulated ChERVs after avian viruses (ALV-J, AIV, MDV) infection, and the reverse is true after a bacterial (APEC) infection (Fig. 2). HERV-K expression could be increased by HIV-1 infection in various cell lines and in vivo [44, 45]. In addition, influenza A virus infection enhanced HERVW expression in cell culture [46]. Our results appear to be the opposite, and more of ChERVs expression levels were inhibited after virus infection in vivo. Indeed, ERVs have been found to be widely involved in the virus-host interaction process, and reports of ERVs involvement in bacterial infections are rare $[47,48]$. Our results suggested that ChERVs might play different functions after viral and bacterial infections in chicken. Given that ERV transcriptional activation will be different depend on different disease conditions and different tissues [49-51], this makes it difficult for us to analyze the differences among the four ChERV transcriptomes in this study. Although we found differences in the four ChERV transcriptomes, it is difficult to elucidate the reasons for these differences because these results come from different pathogens, different tissues, and different infection time points. In future study, we can set a specific tissue and infection time point, and then we can better analyze the differences in ChERV transcriptomes caused by different pathogenic microorganisms. Obviously, in this study, it makes more sense to analyze the intersection of the four ChERV transcriptomes. Coincidently, only one significantly different expressed ChERV, namely ChERV-3, simultaneously existed in 4 ChERV transcriptomes. Interestingly, ChERV-3 expression was significantly down-regulated in all 4 ChERV transcriptomes. These results suggested that ChERV-3 might be involved in the host's responses to ALV-J, AIV, MDV and APEC infections in an indirect way.

Within the range of experimental materials we have, only ALV-J was selected to verify the interaction between pathogen infection and ChERV-3. We demonstrated that the expression of ChERV-3 envelope protein was inhibited after ALV-J infection in vitro experiment. Although ChERV-3 envelope protein could induce the expression of ISGs, it has no effect on ALV-J replication. Similar results have been found in the study of chicken endogenous virus ev21[52]. ISGs have been shown to have a wide range of antiviral effects [53]. Our previous researches also proved that some chicken ISGs can inhibit ALV-J replication, such as $\mathrm{CH} 25 \mathrm{H}$ and $\mathrm{Mx}[54,55]$. Thus, the expression of ERVs could produce proteins and nucleic acids, which would induce innate immune response $[48,56]$. A recent study also showed that a long-nocoding RNA derived from chicken endogenous retroviruses could trigger anti-ALV-J innate immunity by activating ISGs [21]. Accordingly, we make a hypothesis like that proteins and nucleic acids produced by ChERV-3 trigger host innate immune responses (Fig. 6A, B), which would induce ISGs production to inhibit ALV-J, AIV, MDV and APEC infection (Fig. 6C). To escape this antagonism, ALV-J, AIV, MDV and APEC have to restrict ChERV-3 expression through some unknown mechanism (Fig. 6D). Unfortunately, the specific mechanism of each link is still unclear and needs further research.

\section{Conclusions}

In summary, 436 full-length ERVs were identified in the chicken reference genome Gallus_gallus-5.0. We further analyzed ChERV transcriptome in chicken tissues after ALV-J, AIV, MDV and APEC infection. 
ChERV-3 was all significantly down-regulated in 4 ChERV transcriptomes after ALV-J, AIV, MDV and APEC infection. The envelope protein of ChERV-3 can induce the expression of host ISGs, but appears to have no influence on the replication of ALV-J. To our knowledge, this study is the first systematic analysis of the expression profile of chicken endogenous retroviruses after avian pathogens infection.

\section{Declarations}

\section{Acknowledgments}

Thanks are due to Gene Denovo Corp. for its help in bioinformatics analysis.

\section{Authors' contributions}

MF and MD participated in the design of the study, performed the experiments, collected and analyzed data, and drafted the manuscript. TX helped with the preparation of primary chicken embryo fibroblasts and qPCR experiments. XZ participated in the design, coordination of the study and revising the manuscript. All authors read and approved the final manuscript.

\section{Funding}

This work was supported by the National Natural Science Foundation of China (31970540) and the National Natural Science Foundation Youth Fund of China (31801030, 31802174).

\section{Availability of data and materials}

All data generated or analyzed during this study are included in this published article and its supplementary information files.

\section{Ethics approval and consent to participate}

All procedures involving chick embryo were approved by the South China Agriculture University Institutional Animal Care and Use Committee.

\section{Consent for publication}

All of the authors have approved the final version of the manuscript, agree with this submission to Journal of Animal Science and Biotechnology.

\section{Competing interests}

The authors declare that they have no conflicts of financial interest.

\section{References}


1. Gifford R, Tristem M. The evolution, distribution and diversity of endogenous retroviruses, Virus genes. (2003) 26:291-315.

2. Huda A, Polavarapu N, Jordan IK, McDonald JF. Endogenous retroviruses of the chicken genome, Biology direct. (2008) 3:9.

3. Imakawa K, Nakagawa S, Kusama K. Placental Development and Endogenous Retroviruses, Uirusu. (2016) 66:1-10.

4. Alcazer V, Bonaventura P, Depil S. Human Endogenous Retroviruses (HERVs): Shaping the Innate Immune Response in Cancers, Cancers. (2020) 12.

5. Colmegna I, Garry RF. Role of endogenous retroviruses in autoimmune diseases. Infect Dis Clin N Am. 2006;20:913-29.

6. Katzourakis A, Aswad A. Evolution: Endogenous Viruses Provide Shortcuts in Antiviral Immunity, Current biology: CB. (2016) 26:R427-429.

7. Prudhomme S, Bonnaud B, Mallet F. Endogenous retroviruses and animal reproduction, Cytogenetic and genome research. (2005) 110:353-364.

8. Weiss RA. The discovery of endogenous retroviruses, Retrovirology. (2006) 3:67.

9. Wang Z, Qu L, Yao J, Yang X, Li G, Zhang Y, Li J, Wang X, Bai J, Xu G, Deng X, Yang N, Wu C. An EAVHP insertion in 5' Flanking region of SLCO1B3 causes blue eggshell in the chicken, PLoS genetics. (2013) 9:e1003183.

10. Wragg D, Mwacharo JM, Alcalde JA, Wang C, Han JL, Gongora J, Gourichon D, Tixier-Boichard M, Hanotte O. Endogenous retrovirus EAV-HP linked to blue egg phenotype in Mapuche fowl, PloS one. (2013) 8:e71393.

11. Chang CM, Coville JL, Coquerelle G, Gourichon D, Oulmouden A, Tixier-Boichard M. Complete association between a retroviral insertion in the tyrosinase gene and the recessive white mutation in chickens. BMC Genomics. 2006;7:19.

12. Elferink MG, Vallee AA, Jungerius AP, Crooijmans RP, Groenen MA. Partial duplication of the PRLR and SPEF2 genes at the late feathering locus in chicken. BMC Genomics. 2008;9:391.

13. Li J, Davis BW, Jern P, Dorshorst BJ, Siegel PB, Andersson L. Characterization of the endogenous retrovirus insertion in CYP19A1 associated with henny feathering in chicken, Mobile DNA. (2019) 10:38.

14. Crittenden LB, Fadly AM, Smith EJ. Effect of endogenous leukosis virus genes on response to infection with avian leukosis and reticuloendotheliosis viruses, Avian diseases. (1982) 26:279-294.

15. Crittenden LB, Smith EJ, Fadly AM. Influence of endogenous viral (ev) gene expression and strain of exogenous avian leukosis virus (ALV) on mortality and ALV infection and shedding in chickens, Avian diseases. (1984) 28:1037-1056.

16. Gavora JS, Spencer JL, Benkel B, Gagnon C, Emsley A, Kulenkamp A. Endogenous viral genes influence infection with avian leukosis virus, Avian pathology: journal of the W.V.P.A. (1995) 24:653664. 
17. Hu X, Zhu W, Chen S, Liu Y, Sun Z, Geng T, Song C, Gao B, Wang X, Qin A, Cui H. Expression patterns of endogenous avian retrovirus ALVE1 and its response to infection with exogenous avian tumour viruses. Archives of virology. 2017;162:89-101.

18. Cohen CJ, Lock WM, Mager DL. Endogenous retroviral LTRs as promoters for human genes: a critical assessment. Gene. 2009;448:105-14.

19. Chen Y, Yan Q, Zhou P, Li S, Zhu F. HERV-W env regulates calcium influx via activating TRPC3 channel together with depressing DISC1 in human neuroblastoma cells. J Neurovirol. 2019;25:10113.

20. Mangeney M, de Parseval N, Thomas G, Heidmann T. The full-length envelope of an HERV-H human endogenous retrovirus has immunosuppressive properties. J Gen Virol. 2001;82:2515-8.

21. Chen S, Hu X, Cui IH, Wu S, Dou C, Liu Y, Sun Z, Xue S, Geng T, Liu Z, Qin A, Cui H. An endogenous retroviral element exerts an antiviral innate immune function via the derived IncRNA Inc-ALVE1-AS1, Antiviral research. (2019) 170:104571.

22. Zhou B, Qi F, Wu F, Nie H, Song Y, Shao L, Han J, Wu Z, Saiyin H, Wei G, Wang P, Ni T, Qian F. Endogenous Retrovirus-Derived Long Noncoding RNA Enhances Innate Immune Responses via Derepressing RELA Expression, mBio. (2019) 10.

23. Li Z, Chen B, Feng M, Ouyang H, Zheng M, Ye Q, Nie Q, Zhang X. MicroRNA-23b Promotes Avian Leukosis Virus Subgroup J (ALV-J) Replication by Targeting IRF1, Scientific reports. (2015) 5:10294.

24. Smith J, Smith N, Yu L, Paton IR, Gutowska MW, Forrest HL, Danner AF, Seiler JP, Digard P, Webster $R G$, Burt DW. A comparative analysis of host responses to avian influenza infection in ducks and chickens highlights a role for the interferon-induced transmembrane proteins in viral resistance. BMC Genomics. 2015;16:574.

25. Dang L, Teng M, Li HW, Li HZ, Ma SM, Zhao P, Li XJ, Deng RG, Zhang GP, Luo J. Dynamic Changes in the Splenic Transcriptome of Chickens during the Early Infection and Progress of Marek's Disease. Scientific reports. 2017;7:11648.

26. Sun H, Liu P, Nolan LK, Lamont SJ. Novel Pathways Revealed in Bursa of Fabricius Transcriptome in Response to Extraintestinal Pathogenic Escherichia coli (ExPEC) Infection, PloS one. (2015) 10:e0142570.

27. Ellinghaus D, Kurtz S, Willhoeft U. LTRharvest, an efficient and flexible software for de novo detection of LTR retrotransposons. BMC Bioinform. 2008;9:18.

28. Steinbiss S, Willhoeft U, Gremme G, Kurtz S. Fine-grained annotation and classification of de novo predicted LTR retrotransposons, Nucleic acids research. (2009) 37:7002-7013.

29. Zhuo X, Rho M, Feschotte C. Genome-wide characterization of endogenous retroviruses in the bat Myotis lucifugus reveals recent and diverse infections. Journal of virology. 2013;87:8493-501.

30. Kim D, Pertea G, Trapnell C, Pimentel H, Kelley R, Salzberg SL. TopHat2: accurate alignment of transcriptomes in the presence of insertions, deletions and gene fusions, Genome biology. (2013) 14:R36. 
31. Trapnell C, Roberts A, Goff L, Pertea G, Kim D, Kelley DR, Pimentel H, Salzberg SL, Rinn JL, Pachter L. Differential gene and transcript expression analysis of RNA-seq experiments with TopHat and Cufflinks, Nature protocols. (2012) 7:562-578.

32. Li B, Dewey CN. RSEM: accurate transcript quantification from RNA-Seq data with or without a reference genome. BMC Bioinform. 2011;12:323.

33. Himly M, Foster DN, Bottoli I, lacovoni JS, Vogt PK. The DF-1 chicken fibroblast cell line: transformation induced by diverse oncogenes and cell death resulting from infection by avian leukosis viruses. Virology. 1998;248:295-304.

34. Dai M, Feng M, Liu D, Cao W, Liao M. Development and application of SYBR Green I real-time PCR assay for the separate detection of subgroup $\mathrm{J}$ Avian leukosis virus and multiplex detection of avian leukosis virus subgroups A and B, Virology journal. (2015) 12:52.

35. Gao H, Zhao L, Wang H, Xie E, Wang X, Wu Q, Yu Y, He X, Ji H, Rink L, Min J, Wang F. Metal transporter Slc39a10 regulates susceptibility to inflammatory stimuli by controlling macrophage survival. Proc Natl Acad Sci USA. 2017;114:12940-5.

36. Walsh MC, Lee J, Choi Y. Tumor necrosis factor receptor- associated factor 6 (TRAF6) regulation of development, function, and homeostasis of the immune system, Immunological reviews. (2015) 266:72-92.

37. Moore AJ, In TS, Trotman-Grant A, Yoganathan K, Montpellier B, Guidos CJ, Zuniga-Pflucker JC, Anderson MK. A key role for IL-7R in the generation of microenvironments required for thymic dendritic cells, Immunology and cell biology. (2017) 95:933-942.

38. Mommert M, Tabone O, Oriol G, Cerrato E, Guichard A, Naville M, Fournier P, Volff JN, Pachot A, Monneret G, Venet F, Brengel-Pesce K, Textoris J, Mallet F., LTR-retrotransposon transcriptome modulation in response to endotoxin-induced stress in PBMCs, BMC genomics. (2018) 19:522.

39. Bolisetty M, Blomberg J, Benachenhou F, Sperber G, Beemon K. Unexpected diversity and expression of avian endogenous retroviruses, mBio. (2012) 3:e00344-00312.

40. Stoye JP. Studies of endogenous retroviruses reveal a continuing evolutionary saga, Nature reviews. Microbiology. 2012;10:395-406.

41. Crichton JH, Dunican DS, Maclennan M, Meehan RR, Adams IR. Defending the genome from the enemy within: mechanisms of retrotransposon suppression in the mouse germline, Cellular and molecular life sciences: CMLS. (2014) 71:1581-1605.

42. Rowe HM, Trono D. Dynamic control of endogenous retroviruses during development, Virology. (2011) 411:273-287.

43. Jern P, Coffin JM. Effects of retroviruses on host genome function. Annual review of genetics. 2008;42:709-32.

44. Contreras-Galindo R, Lopez P, Velez R, Yamamura Y. HIV-1 infection increases the expression of human endogenous retroviruses type K (HERV-K) in vitro, AIDS research and human retroviruses. (2007) 23:116-122. 
45. Bhardwaj N, Maldarelli F, Mellors J, Coffin JM. HIV-1 infection leads to increased transcription of human endogenous retrovirus HERV-K (HML-2) proviruses in vivo but not to increased virion production. Journal of virology. 2014;88:11108-20.

46. Nellaker $\mathrm{C}$, Yao Y, Jones-Brando L, Mallet F, Yolken RH, Karlsson H, Transactivation of elements in the human endogenous retrovirus W family by viral infection, Retrovirology. (2006) 3:44.

47. Leung A, Trac C, Kato H, Costello KR, Chen Z, Natarajan R, Schones DE. LTRs activated by EpsteinBarr virus-induced transformation of B cells alter the transcriptome, Genome research. (2018) 28:1791-1798.

48. Aswad A, Katzourakis A. Paleovirology and virally derived immunity, Trends in ecology \& evolution. (2012) 27:627-636.

49. Perot P, Mugnier N, Montgiraud C, Gimenez J, Jaillard M, Bonnaud B, Mallet F. Microarray-based sketches of the HERV transcriptome landscape, PloS one. (2012) 7:e40194.

50. Conley AB, Piriyapongsa J, Jordan IK. Retroviral promoters in the human genome, Bioinformatics. (2008) 24:1563-1567.

51. Prudencio M, Gonzales PK, Cook CN, Gendron TF, Daughrity LM, Song Y, Ebbert MTW, van Blitterswijk M, Zhang YJ, Jansen-West K, Baker MC, DeTure M, Rademakers R, Boylan KB, Dickson DW, Petrucelli L, Link CD. Repetitive element transcripts are elevated in the brain of C9orf72 ALS/FTLD patients, Human molecular genetics. (2017) 26:3421-3431.

52. Feng M, Tan Y, Dai M, Li Y, Xie T, Li H, Shi M, Zhang X. Endogenous Retrovirus ev21 Dose Not Recombine with ALV-J and Induces the Expression of ISGs in the Host, Frontiers in cellular and infection microbiology. (2016) 6:140.

53. Schoggins JW, Rice CM. Interferon-stimulated genes and their antiviral effector functions. Current opinion in virology. 2011;1:519-25.

54. Xie T, Feng M, Dai M, Mo G, Ruan Z, Wang G, Shi M, Zhang X, Cholesterol-25-hydroxylase Is a Chicken ISG That Restricts ALV-J Infection by Producing 25-hydroxycholesterol, Viruses. (2019) 11.

55. Feng M, Zhang N, Xie T, Ren F, Cao Z, Zeng X, Swevers L, Zhang X, Sun J. Chichen type III interferon produced by silkworm bioreactor induces ISG expression and restricts ALV-J infection in vitro, Applied microbiology and biotechnology. (2019) 103:8473-8483.

56. Hurst TP, Magiorkinis G. Activation of the innate immune response by endogenous retroviruses. J Gen Virol. 2015;96:1207-18.

\section{Figures}


$\mathbf{A}$
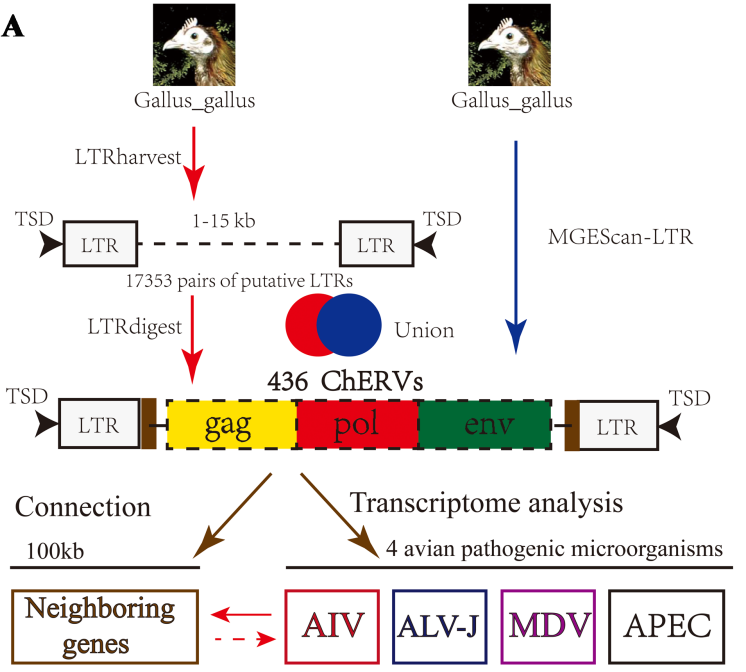

C

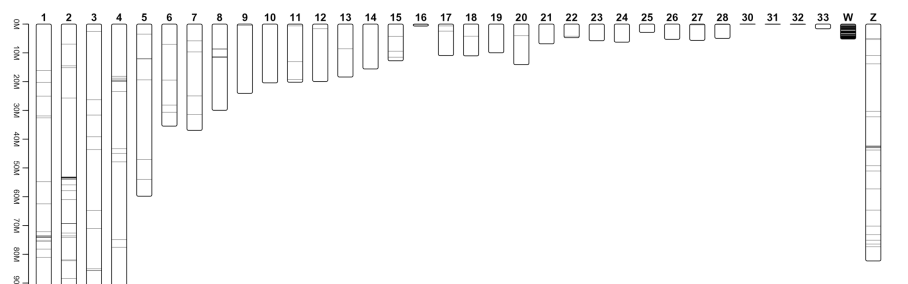

D

Biological Process

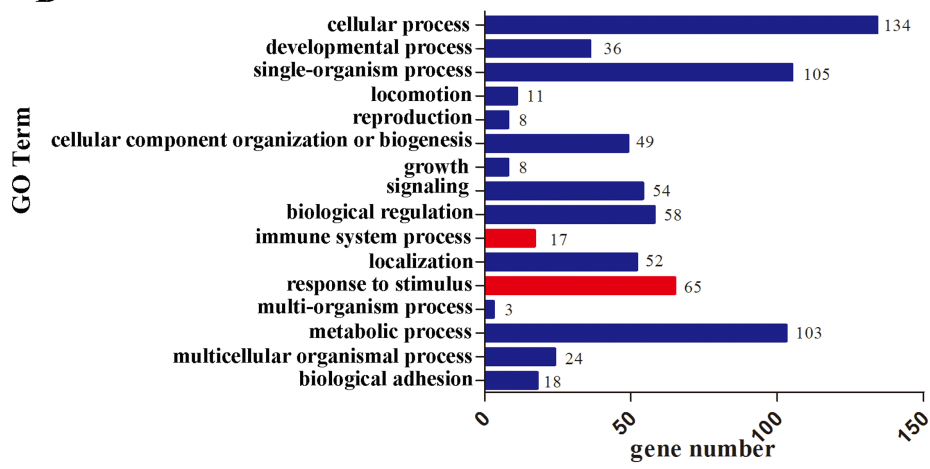

\section{B}

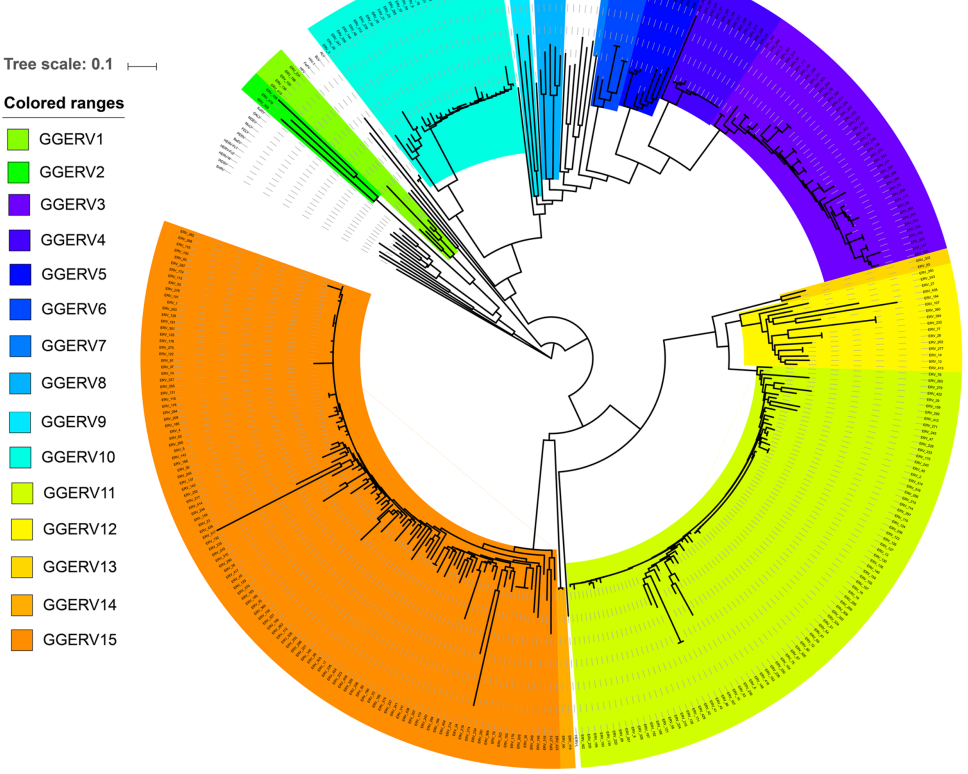

$\mathbf{E}$

Top 20 of Pathway Enrichment

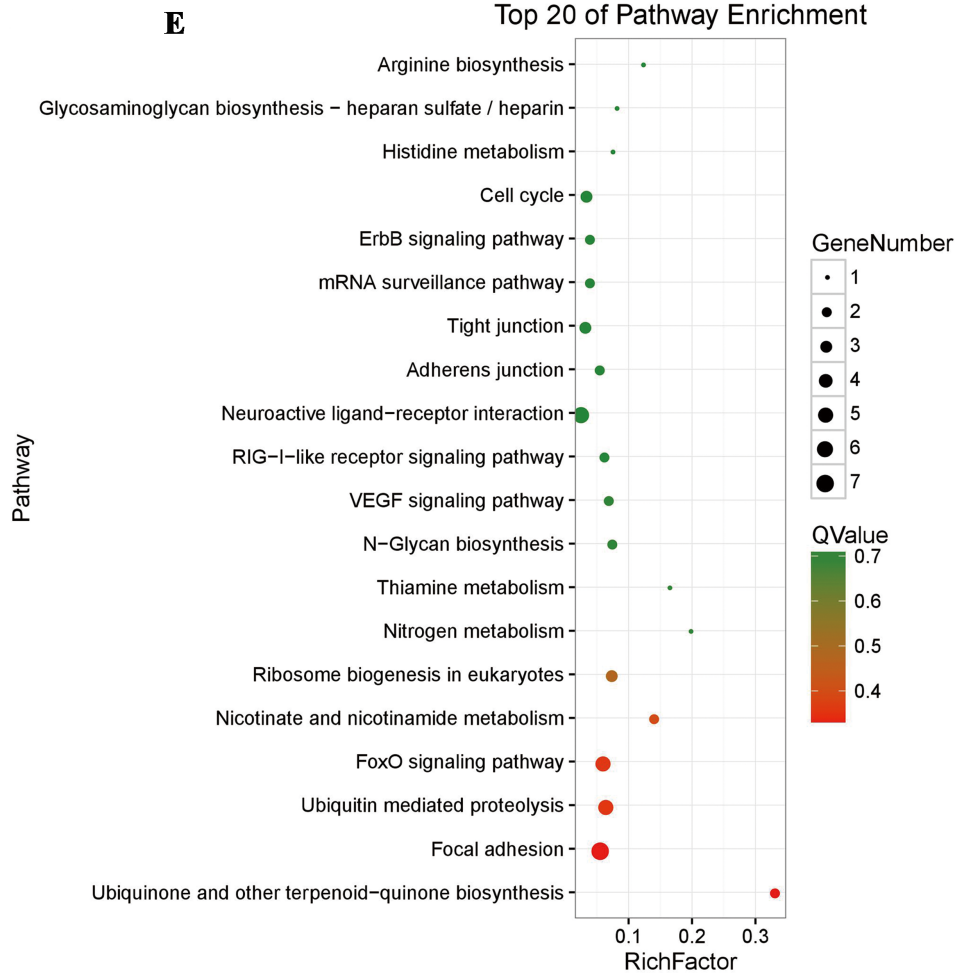

Figure 1

Analysis of ERVs and their neighboring genes in the chicken genome. (A) Summary of the strategy used to analyze ChERVs in this study. We identified 17,353 LTR candidates using LTRharvest, and all of them were annotated using LTRdigest. We also used the independent pipeline MGEScan-LTR to identify potential complete ChERVs. By combining the two independent pipelines, a total of 436 potential complete ChERVs were identified in chicken genome Gallus_gallus-5.0. We further analyzed their neighboring genes located within $100 \mathrm{~kb}$ of upstream and downstream of ChERVs. Moreover, ChERVs transcriptomes were analyzed in chicken after AIV, ALV-J, MDV and APEC infection. (B) Phylogenetic tree 
of ChERVs based on the alignment of the RT region. A total of 384 ChERVs contained an RT domain with sufficient conservation for confident alignment prior to phylogenetic analysi. Fifteen families of ChERVs are illustrated with different color. Known exogenous and endogenous retroviruses were referenced from the reported paper [29] and no color overlay. Topology was based on the neighbor-joining method with 1,000 bootstraps. (C) Distribution of ChERVs on chicken chromosomes. The lines on the chromosomes represent the approximate location of the ChERVs. A large number of ChERVs are integrated on the W chromosome. The $\mathrm{W}$ chromosome is enlarged to show the location of ChERVs. (D, E) GO and KEGG analysis of the neighboring genes of ChERVs. (D) The representative biological process (BP) in Gene Ontology (GO) terms of neighboring genes of ChERVs. Go terms including "immune system process" and "response to stimulus" were shown red. (E) The neighboring genes of ChERVs that were enriched in KEGG pathways. Genes located $\sim 100 \mathrm{~kb}$ upstream and downstream of ChERVs were identified as "neighboring" genes. 

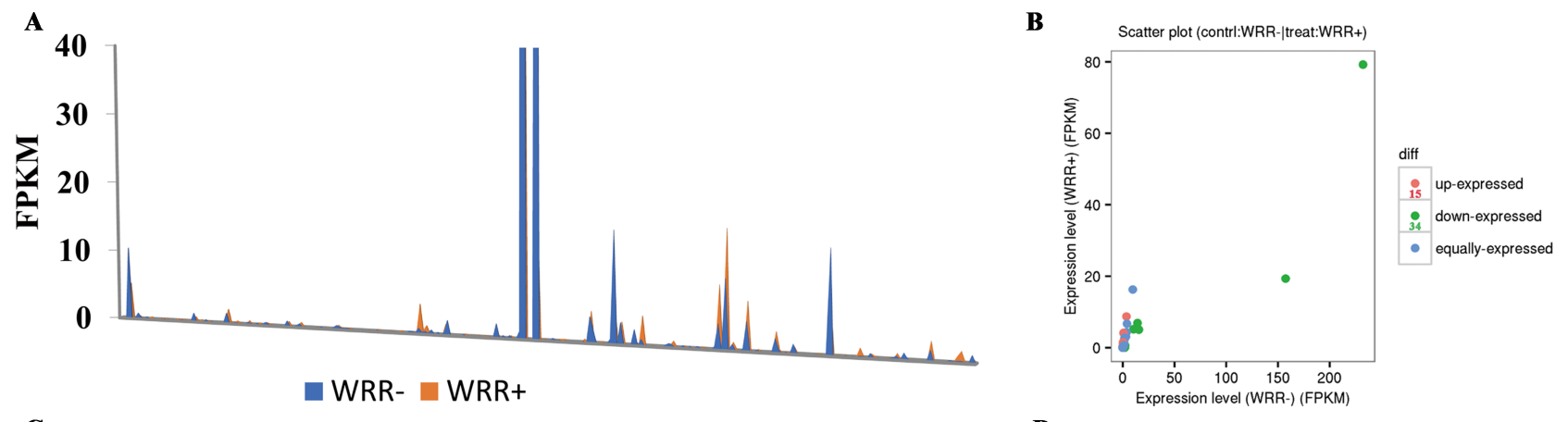

$\mathbf{C}$
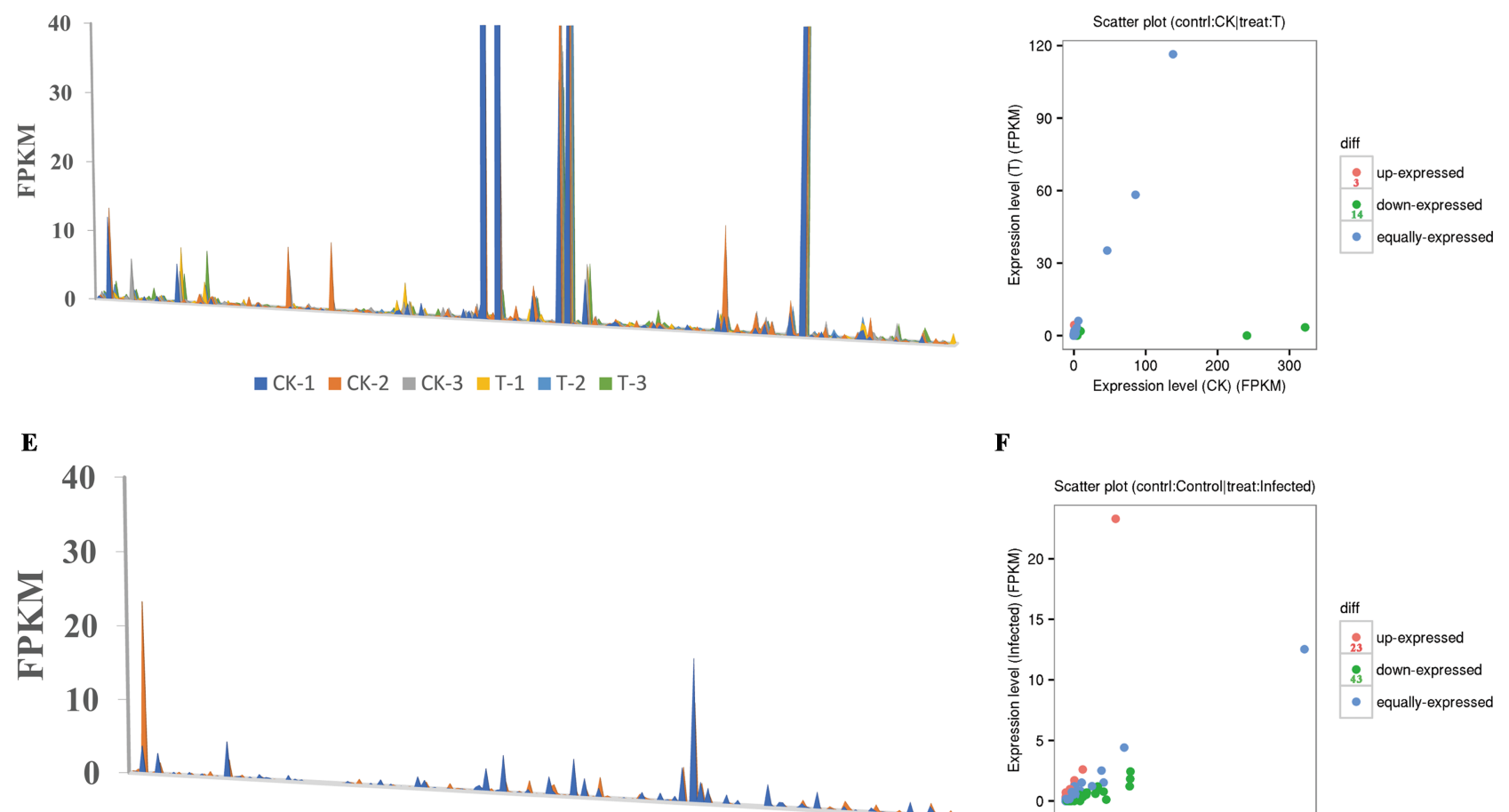

- Control $\mathbf{\text { anfected }}$

F

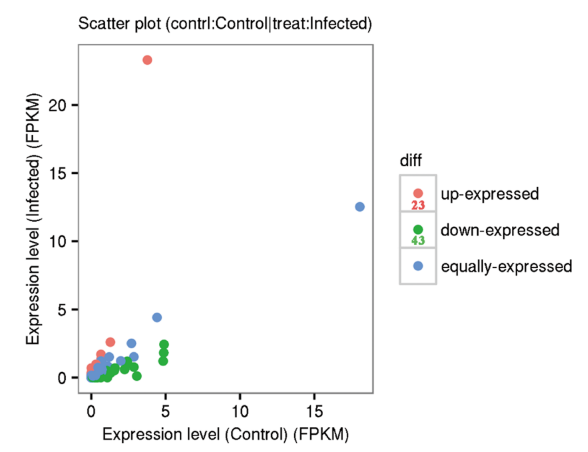

G

H
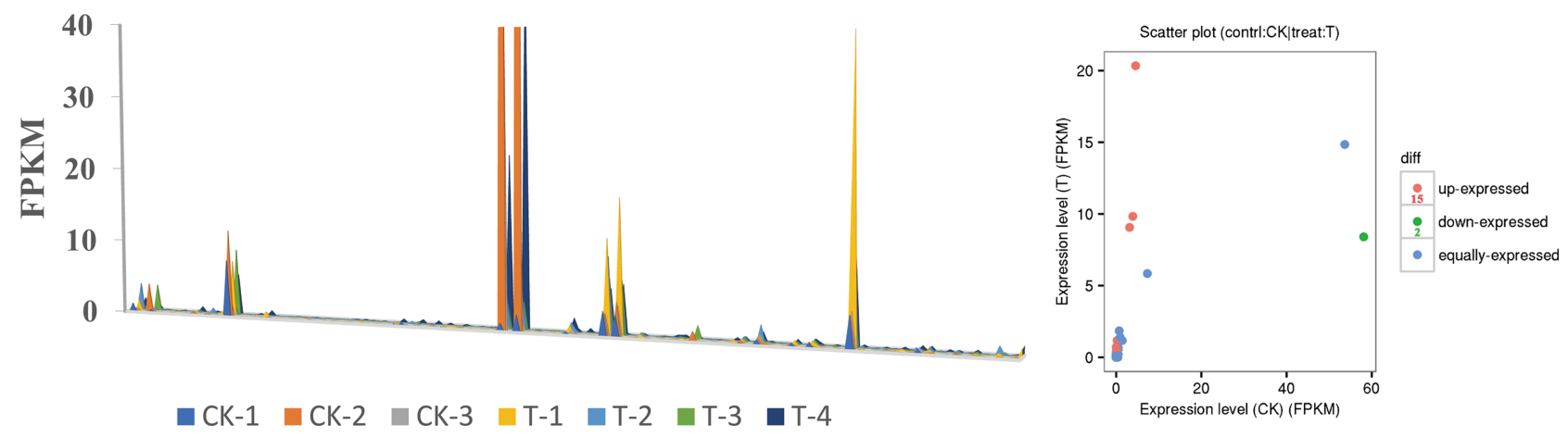

Figure 4

Expression of ChERVs in ALV-J, AIV, MDV and APEC infected samples and corresponding uninfected samples. RNA-seq data from ALV-J (A) [23], AIV (C) [24], MDV (E) [25] and APEC-infected chicken (F) [26] and corresponding uninfected controls were aligned using TopHat and transcripts were constructed using Cufflinks. The number of fragments per kilobase per million reads (FPKM) was plotted against the ChERVs. Scatter plot of differentially expressed ChERVs between ALV-J (B), AIV (D), MDV (F) and APEC- 
infected chicken $(\mathrm{H})$ and corresponding uninfected controls. The red spots represent significantly upregulated ChERVs. The green spots represent significantly down-regulated ChERVs. The blue spots indicate absence of significantly different expression.

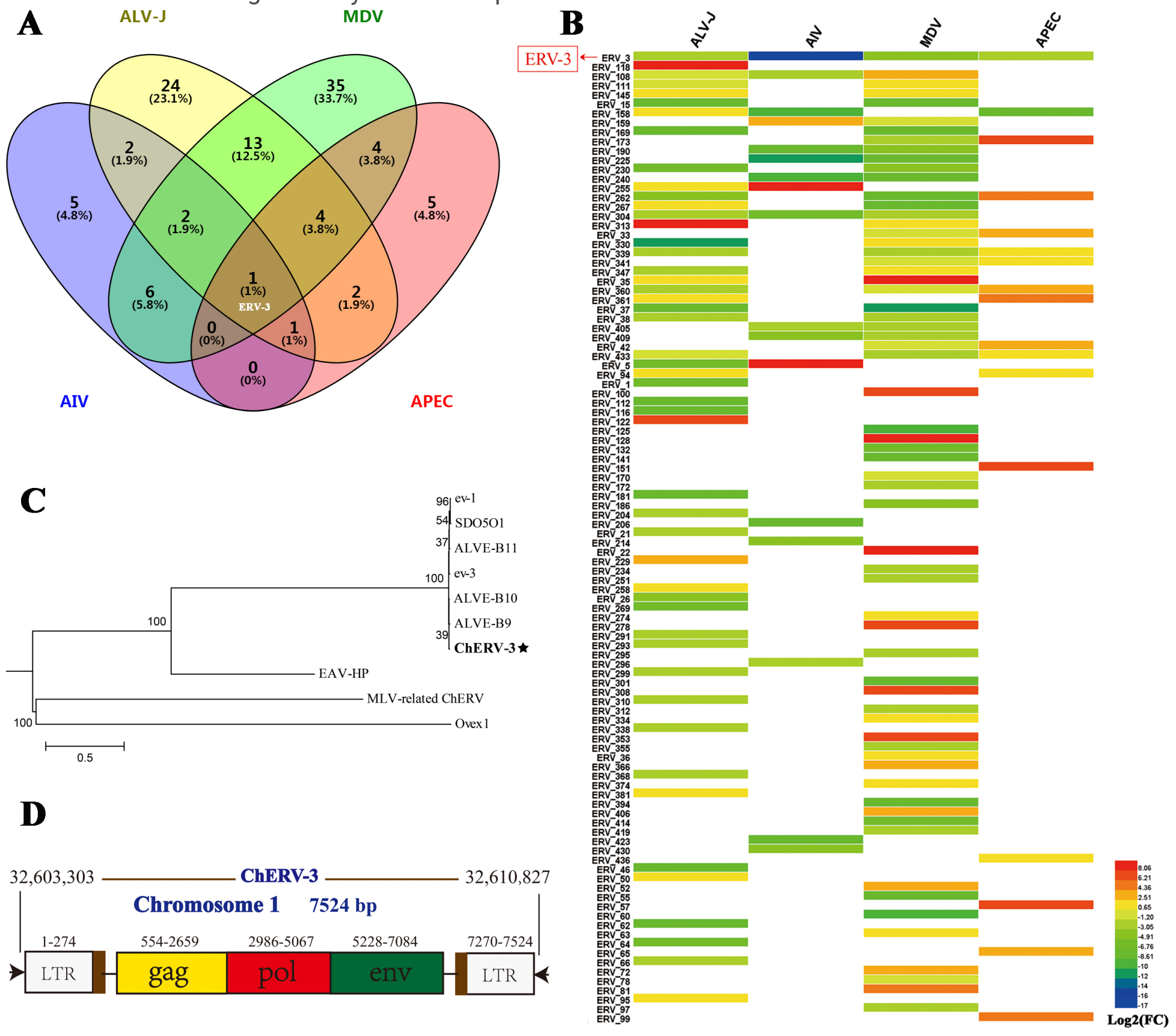

Figure 7

Analysis of differentially expressed ChERVs in ALV-J, AIV, MDV and APEC infection. (A) Venn diagrams of significantly different expressed ChERVs in ALV-J (yellow), AIV (blue), MDV (green) and APEC (red)infected chicken. The intersection of the four sets of data has only one ChERV, which is ChERV-3. (B) Heatmap of significantly different expressed ChERVs in ALV-J, AIV, MDV and APEC-infected chicken. Heatmap showed that ChERV-3 was significantly down-regulated after ALV-J, AIV, MDV and APEC infection. (C) Phylogenetic relationship of full-length ChERV-3 to reference chicken endogenous virus ev1 (GenBank: AY013303.1), SD0501 (EF467236.1), ALVE-B11 (KC610517.1), ev3 (AY013304.1), ALVE-B10 
(KC610516.1), ALVE-B9 (KC610515.1), EAV-HP (NC_005947.1), MLV-related ChERV (DQ280312.2), Ovex1 (FJ406461.1). (D) The position of ChERV-3 on the chromosome 1 and its genomic structure.
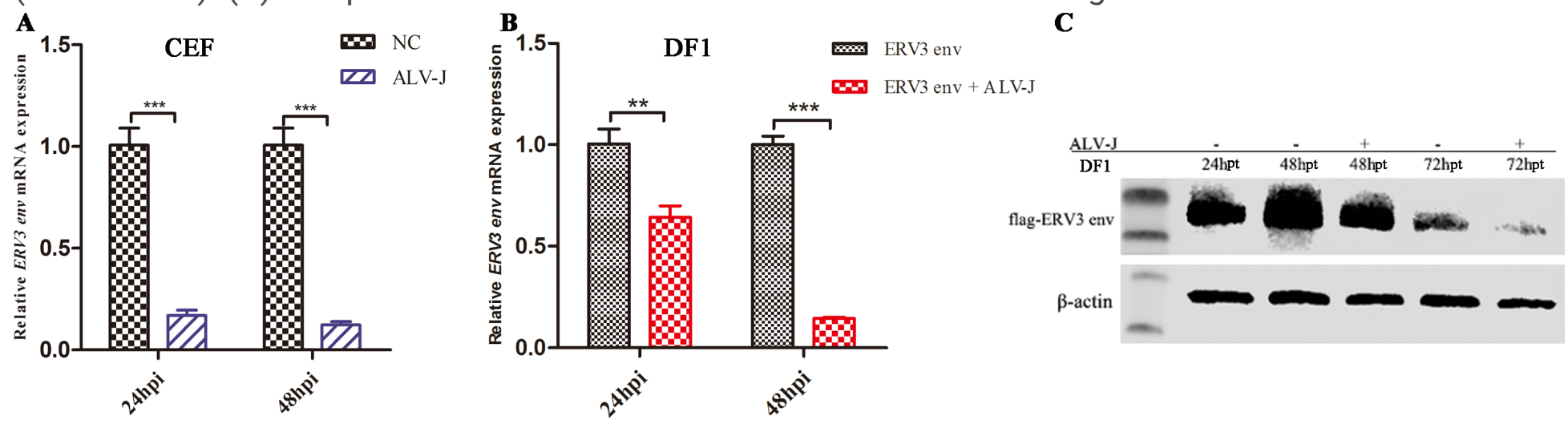

Figure 10

Effect of ALV-J infection on ChERV-3 expression. (A) Primary chicken embryo fibroblasts (CEF) were infected by ALV-J (SCAU-HN06, 105 TCID 50/mL). Uninfected CEF was used as control. qPCR was used to detect the endogenous expression of ChERV-3 env gene after infection at 24 and $48 \mathrm{~h}$ post infection (hpi). (B, C) DF1 cells were cultured in 24-well plates and transfected with $0.75 \mu \mathrm{g}$ pcDNA3.1-ERV3env plasmid. $24 \mathrm{~h}$ later, the transfected DF1 cells were infected with $105 \mathrm{TCID} 50 / \mathrm{mL}$ of ALV-J strain SCAU-HN06. Uninfected DF1 cells transfected with pcDNA3.1-ERV3env plasmid were used as control. The exogenous expression of ChERV3 env mRNA was analyzed by qPCR (B) and the envelope protein was analyzed by Western blot (C) using flag antibody at 24 and $48 \mathrm{hpi}$. Data are representative of three independent experiments. ${ }^{* *} \mathrm{p}<0.01,{ }^{* * *} \mathrm{p}<0.001$. Error bars indicate SEM.
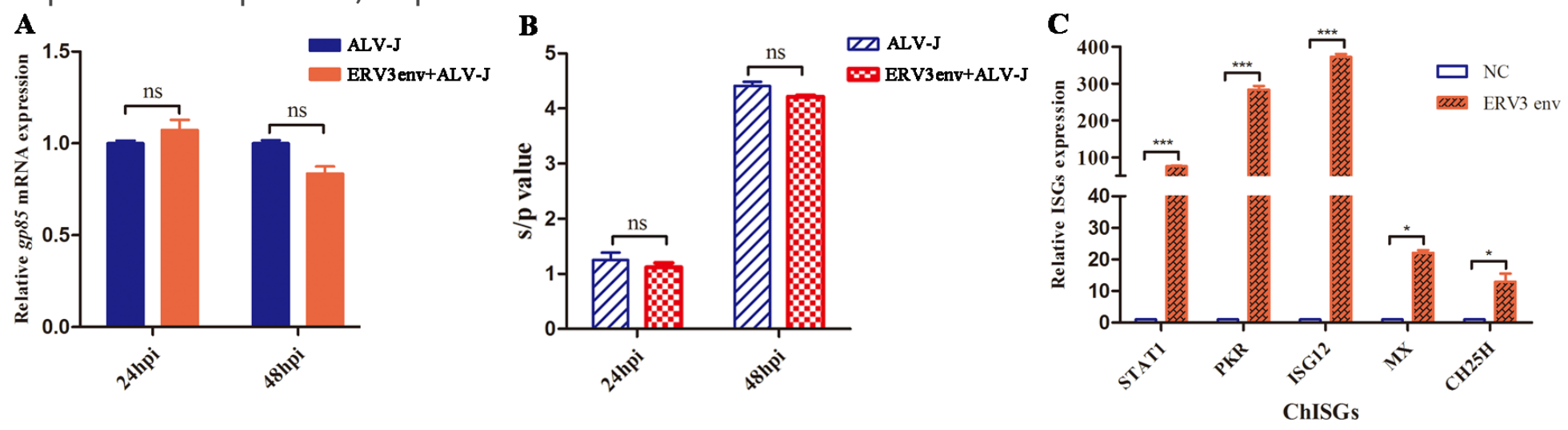

Figure 13

Analysis of ALV-J infection and ISGs expression after ChERV-3 env overexpression. (A, B) DF1 cells were transfected with $0.75 \mu \mathrm{g}$ pcDNA3.1-ERV3env plasmid and pcDNA3.1 empty vector (NC), respectively. These transfected DF1 cells were infected with $105 \mathrm{TCID50/mL}$ of ALV-J strain SCAU-HN06 at $24 \mathrm{~h}$ post transfection. qPCR (A) was employed to detecting the production of ALV-J in mRNA level using cDNA samples extracted from cells. The cell supernatants were tested for ALV group-specific antigen (p27) by ELISA (B). $s / p=$ (Sample Mean-Kit Negative Control Mean)/(Kit Positive Control Mean-Kit Negative Control Mean). (C) DF1 cells were transfected with $0.75 \mu \mathrm{g}$ pcDNA3.1-ERV3env plasmid and pcDNA3.1 
empty vector, respectively. $24 \mathrm{~h}$ later, chicken interferon-stimulated genes (ISGs) including STAT1, EIF2AK2(PKR), ISG12, Mx and CH25H were detected by qPCR. ${ }^{*} \mathrm{p}<0.05$, ${ }^{\star \star \star} \mathrm{p}<0.001$. ns, not significant. Error bars indicate SEM. All experiments were performed in triplicate.

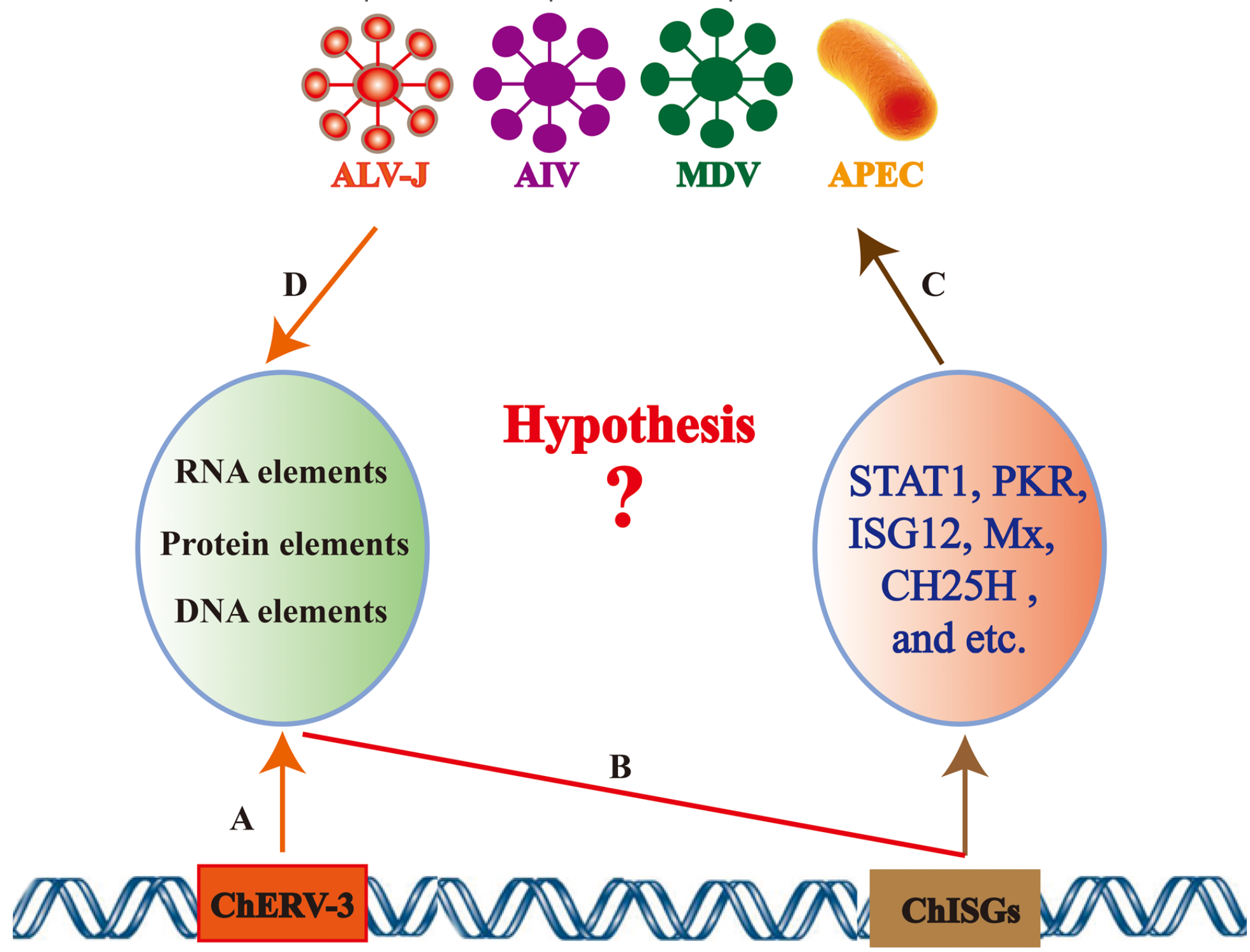

Figure 16

Hypothesis that ChERV-3 participates in host resistance to avian pathogenic microorganism infection. (A) Protein and nucleic acid elements might be produced by ChERV-3 during transcription and translation. (B) Proteins and nucleic acids elements produced by ChERV-3 might trigger host innate immune responses that induces ISGs production. (C) ISGs induced by ChERV-s could inhibit ALV-J, AIV, MDV and APEC infection. (D) To escape the antagonistic effects of ISGs, ALV-J, AIV, MDV and APEC might restrict ChERV3 expression through some unknown mechanism.

\section{Supplementary Files}

This is a list of supplementary files associated with this preprint. Click to download. 
- Additionalfile9TableS7.xlsx

- Additionalfile8TableS6.xlsx

- Additionalfile8TableS6.xIsx

- Additionalfile3TableS2.xIsx

- Additionalfile7TableS5.xlsx

- Additionalfile1.docx

- Additionalfile8TableS6.xIsx

- Additionalfile9Tables7.xlsx

- Additionalfile4TableS3.xlsx

- Additionalfile4TableS3.xIsx

- Additionalfile3TableS2.xIsx

- Additionalfile7TableS5.xIsx

- Additionalfile6Figures1.tif

- Additionalfile3Tables2.xlsx

- Additionalfile6Figures1.tif

- Additionalfile9Tables7.xlsx

- Additionalfile1.docx

- Additionalfile2TableS1.xIsx

- Additionalfile5TableS4.xlsx

- Additionalfile1.docx

- Additionalfile2TableS1.xlsx

- Additionalfile5TableS4.xlsx

- Additionalfile4TableS3.xlsx

- Additionalfile5TableS4.xlsx

- Additionalfile2TableS1.xlsx

- Additionalfile6FigureS1.tif

- Additionalfile7TableS5.xlsx 